\title{
Muscle oxidative phosphorylation quantitation using creatine chemical exchange saturation transfer (CrCEST) MRI in mitochondrial disorders
}

\author{
Catherine DeBrosse, ${ }^{1}$ Ravi Prakash Reddy Nanga, ${ }^{1}$ Neil Wilson, ${ }^{1}$ Kevin D'Aquilla, ${ }^{1}$ Mark Elliott, ${ }^{1}$ \\ Hari Hariharan, ${ }^{1}$ Felicia Yan, ${ }^{2}$ Kristin Wade, ${ }^{2}$ Sara Nguyen, ${ }^{2}$ Diana Worsley, ${ }^{2}$ \\ Chevonne Parris-Skeete, ${ }^{2}$ Elizabeth McCormick, ${ }^{3}$ Rui Xiao, ${ }^{4}$ Zuela Zolkipli Cunningham, ${ }^{5}$ \\ Lauren Fishbein, ${ }^{6}$ Katherine L. Nathanson, ${ }^{7,8}$ David R. Lynch, ${ }^{5}$ Virginia A. Stallings,, 10 \\ Marc Yudkoff,, ${ }^{3,10}$ Marni J. Falk,, ${ }^{3,10}$ Ravinder Reddy, ${ }^{1}$ and Shana E. McCormack ${ }^{2,10}$ \\ ${ }^{1}$ Center for Magnetic Resonance and Optical Imaging, Department of Radiology, University of Pennsylvania, Philadelphia, \\ Pennsylvania, USA. ${ }^{2}$ Division of Endocrinology and Diabetes, The Children's Hospital of Philadelphia, ${ }^{3}$ Division of Human \\ Genetics, The Children's Hospital of Philadelphia, Philadelphia, Pennsylvania, USA. ${ }^{4}$ Department of Biostatistics and \\ Epidemiology, Perelman School of Medicine, University of Pennsylvania, Philadelphia, Pennsylvania, USA. ${ }^{5}$ Department \\ of Neurology, The Children's Hospital of Philadelphia, Philadelphia, Pennsylvania, USA. 'Division of Endocrinology, \\ Metabolism, and Diabetes, University of Colorado School of Medicine, Aurora, Colorado, USA. 'Division of Translationa \\ Medicine and Human Genetics, Department of Medicine, Perelman School of Medicine, University of Pennsylvania \\ ${ }^{8}$ Abramson Cancer Center, Perelman School of Medicine, University of Pennsylvania, ${ }^{9}$ Division of Gastroenterology, \\ Hepatology, and Nutrition, The Children's Hospital of Philadelphia, ${ }^{10}$ Department of Pediatrics, Perelman School of \\ Medicine, University of Pennsylvania, Philadelphia, Pennsylvania, USA.
}

Systemic mitochondrial energy deficiency is implicated in the pathophysiology of many age-related human diseases. Currently available tools to estimate mitochondrial oxidative phosphorylation (OXPHOS) capacity in skeletal muscle in vivo lack high anatomic resolution. Muscle groups vary with respect to their contractile and metabolic properties. Therefore, muscle group-specific estimates of OXPHOS would be advantageous. To address this need, a noninvasive creatine chemical exchange saturation transfer (CrCEST) MRI technique has recently been developed, which provides a measure of free creatine. After exercise, skeletal muscle can be imaged with CrCEST in order to make muscle group-specific measurements of OXPHOS capacity, reflected in the recovery rate $(\tau \mathrm{Cr})$ of free $\mathrm{Cr}$. In this study, we found that individuals with genetic mitochondrial diseases had significantly $(P=0.026)$ prolonged postexercise $\tau \mathrm{Cr}$ in the medial gastrocnemius muscle, suggestive of less OXPHOS capacity. Additionally, we observed that lower resting CrCEST was associated with prolonged $\tau \mathrm{PCr}$, with a Pearson's correlation coefficient of $-0.42(P=0.046)$, consistent with previous hypotheses predicting that resting creatine levels may correlate with ${ }^{31} \mathrm{P}$ magnetic resonance spectroscopy-based estimates of OXPHOS capacity. We conclude that CrCEST can noninvasively detect changes in muscle creatine content and OXPHOS capacity, with high anatomic resolution, in individuals with mitochondrial disorders.

Conflict of interest: The authors have declared that no conflict of interest exists.

Submitted: April 25, 2016 Accepted: September 29, 2016 Published: November 3, 2016

Reference information: JCI Insight. 2016;1(18):e88207. doi:10.1172/jci.insight.88207.

\section{Introduction}

Systemic defects in mitochondrial bioenergetics occur in primary mitochondrial disorders, as well as in various age-related human disorders, including diabetes mellitus, cardiovascular disease, Alzheimer's and Parkinson's diseases, and cancer (1). To investigate the role of mitochondrial function in these diseases, it is essential to measure mitochondrial energy production in vivo, ideally with noninvasive tools. Skeletal muscle has been a particular focus of in vivo methodological developments. Individuals with genetic disorders of the mitochondria often have impaired energy production and consequently suffer from exercise intolerance and fatigue (2). Decreased exercise capacity is a result of defects in the mitochondrial respiratory chain, the major ATP-yielding metabolic pathway in the cell. Energy deficiency is most apparent in tissues 
Table 1. Subject characteristics

\begin{tabular}{|c|c|c|}
\hline & Mitochondrial Disease $(n=13)$ & Healthy Volunteers $(n=14)$ \\
\hline Sex ( $\%$ female, $n$ ) & $62(8)$ & $57(8)$ \\
\hline Age (years) & $42.1 \pm 12.9$ & $45.5 \pm 13.7$ \\
\hline BMI $\left(\mathrm{kg} / \mathrm{m}^{2}\right)$ & $26.5 \pm 6.7$ & $25.9 \pm 4.7$ \\
\hline Underweight, $<18.5(\%, n)$ & $8(1)$ & $0(0)$ \\
\hline Normal weight, 18.5-24.9 $(\%, n)$ & $38(5)$ & $43(6)$ \\
\hline Overweight, 25-30 (\%, $n)$ & $31(4)$ & $43(6)$ \\
\hline Obese, >30 $(\%, n)$ & $23(3)$ & $14(2)$ \\
\hline Self-reported Population Ancestry (\% white, $n$ ) & $92(12)$ & $100(14)$ \\
\hline \multicolumn{3}{|l|}{ Laboratory Studies } \\
\hline HgbA1c (\%) & $5.00 \pm 0.28$ & $5.22 \pm 0.30$ \\
\hline Glucose (mg/dL) & $85 \pm 8$ & $90 \pm 9$ \\
\hline Cholesterol, total (mg/dL) & $190 \pm 36$ & $188 \pm 43$ \\
\hline Triglycerides (mg/dL) & $116 \pm 56$ & $121 \pm 75$ \\
\hline HDL (mg/dL) & $52 \pm 12$ & $52 \pm 10$ \\
\hline LDL (mg/dL) & $115 \pm 26$ & $112 \pm 38$ \\
\hline \multicolumn{3}{|l|}{ Self-reported Physical Activity ${ }^{A}$} \\
\hline Total (hrs per wk) & $7.3 \pm 4.5$ & $9.3 \pm 3.3$ \\
\hline Total (MET-hrs per wk) & $16.4 \pm 11.8^{\mathrm{B}}$ & $25.4 \pm 10.5$ \\
\hline Moderate-heavy activity, >3 METs) (hrs per week) & $0.9 \pm 1.3^{B}$ & $2.5 \pm 1.9$ \\
\hline Moderate-heavy activity, >3 METs (MET-hrs per week) & $4.8 \pm 6.4^{B}$ & $11.2 \pm 8.3$ \\
\hline Intentional exercise (hrs per wk) & $0.7 \pm 0.8^{\mathrm{B}}$ & $1.5 \pm 0.9$ \\
\hline Intentional exercise (MET-hrs per week) & $4.0 \pm 4.9$ & $7.2 \pm 3.8$ \\
\hline \multicolumn{3}{|l|}{ Newcastle Mitochondrial Disease Adult Scale ${ }^{\complement}$} \\
\hline 0, asymptomatic $(\%, n)$ & $8(1)^{B}$ & $38(5)$ \\
\hline $1-5$, mild $(\%, n)$ & $38(5)^{\mathrm{B}}$ & $64(9)$ \\
\hline 6-20, moderate $(\%, n)$ & $46(6)^{\mathrm{B}}$ & 0 \\
\hline$>20$, severe $(\%, n)$ & $8(1)^{B}$ & 0 \\
\hline NIH PROMIS Physical Function Scale ${ }^{D}$ & $41.8 \pm 7.6^{\mathrm{E}}$ & $56.8 \pm 2.9$ \\
\hline NIH PROMIS Global Health Scale ${ }^{F}$ & $32.8 \pm 4.2^{\mathrm{E}}$ & $39.5 \pm 3.0$ \\
\hline
\end{tabular}

${ }^{A}$ Self-reported physical activity is estimated over the 4 weeks prior to the study. ${ }^{\mathrm{B}} P<0.05$ for differences between subjects with mitochondrial disease versus healthy volunteers by 2 -sample $t$ test, Wilcoxon rank-sum test, or chi-square test, as appropriate. ${ }^{\mathrm{C}} \mathrm{A}$ higher Newcastle Mitochondrial Disease Adult Scale (NMDAS) score indicates more severe disease. Symptoms are estimated by the subject over the 4 weeks prior to the study. Of note, the NMDAS scale has not been validated in healthy adults but was administered here to indicate the degree of comparable symptom burden. ${ }^{\mathrm{D}} \mathrm{A}$ higher NIH PROMIS physical function score indicates better physical function. ${ }^{E} P<0.001$ for differences between subjects with mitochondrial disease versus healthy volunteers by 2 -sample $t$ test, Wilcoxon rank-sum test, or chi-square test, as appropriate. ${ }^{\mathrm{F} A}$ higher NIH PROMIS global health score indicates better overall health. All statistically significant differences are shown in bold text. HgbA1c, hemoglobin A1c; MET, metabolic equivalent.

with large energy demand, including exercising skeletal muscle. In genetic or acquired defects of muscle oxidative phosphorylation (OXPHOS) capacity, where muscle fiber type is closely coupled to metabolism and function (3-6), the degree of metabolic compromise may therefore be highly variable among muscle groups.

Spectroscopic methods, such as ${ }^{31} \mathrm{P}$ magnetic resonance spectroscopy $\left({ }^{31} \mathrm{P}-\mathrm{MRS}\right)$, have shown deficient OXPHOS and ATP synthesis in individuals with mitochondrial diseases, as reflected by delayed phosphocreatine (PCr) recovery after exercise (7-10). The limited spatial resolution of ${ }^{31} \mathrm{P}-\mathrm{MRS}$ makes the assessment of muscle group-specific OXPHOS capacity more challenging. To address this limitation, methodological innovations in ${ }^{31} \mathrm{P}-\mathrm{MRS}$ are ongoing (11). There is a need for an in vivo technique to measure OXPHOS capacity that provides a snapshot of multiple muscle groups simultaneously. An imaging-based strategy with high spatial resolution would enhance our understanding of the metabolic response to mitochondrial disease in muscle.

A complementary method to ${ }^{31} \mathrm{P}-\mathrm{MRS}$ is creatine chemical exchange saturation transfer (CrCEST) (12), an MRI modality with high spatial resolution that has been utilized to study metabolism in vivo (13). With the CEST technique, metabolites are measured by first selectively saturating protons of interest, in this case, the guanidinium protons of creatine, that are in exchange with the bulk water protons in the 
A
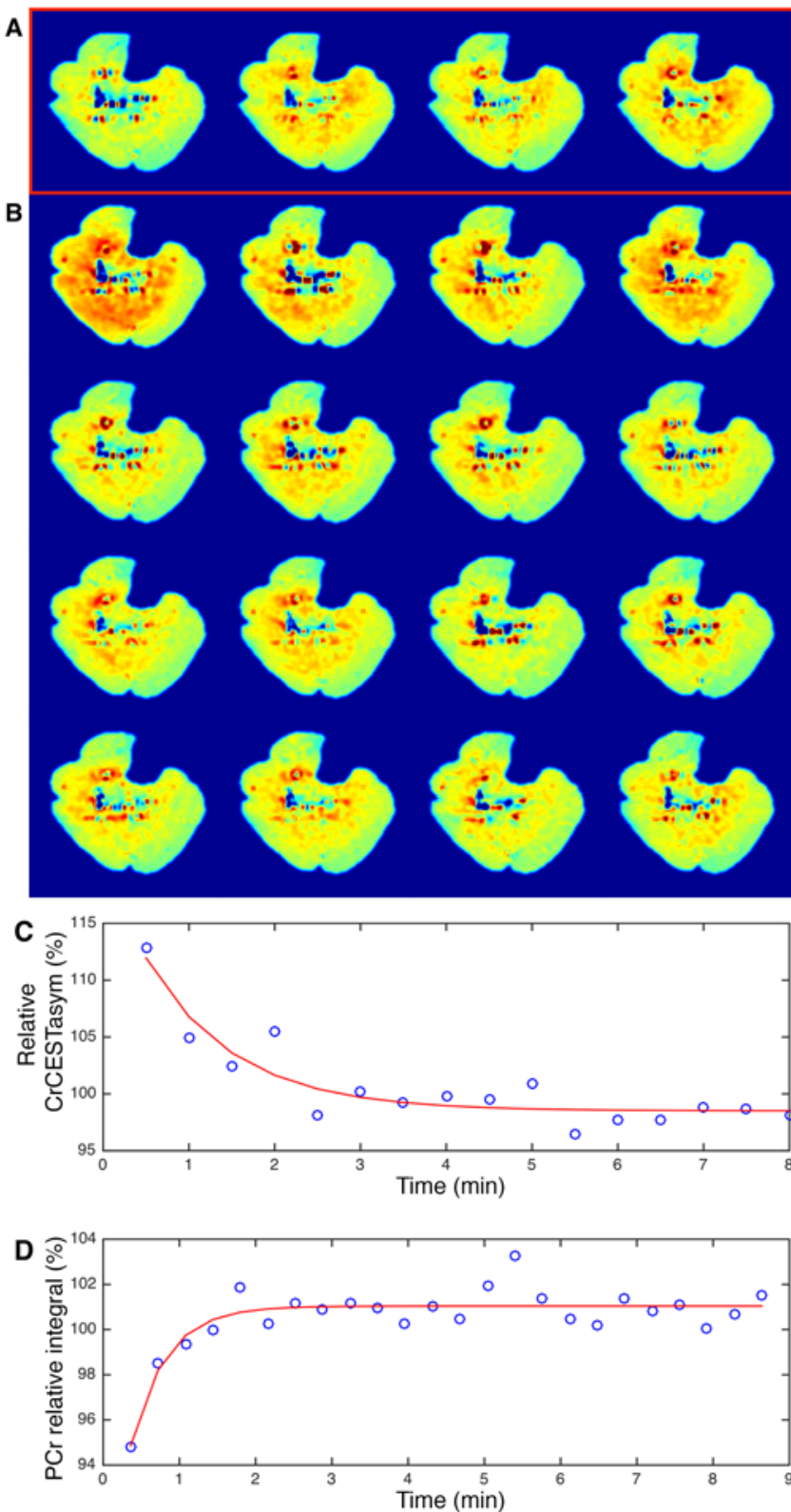
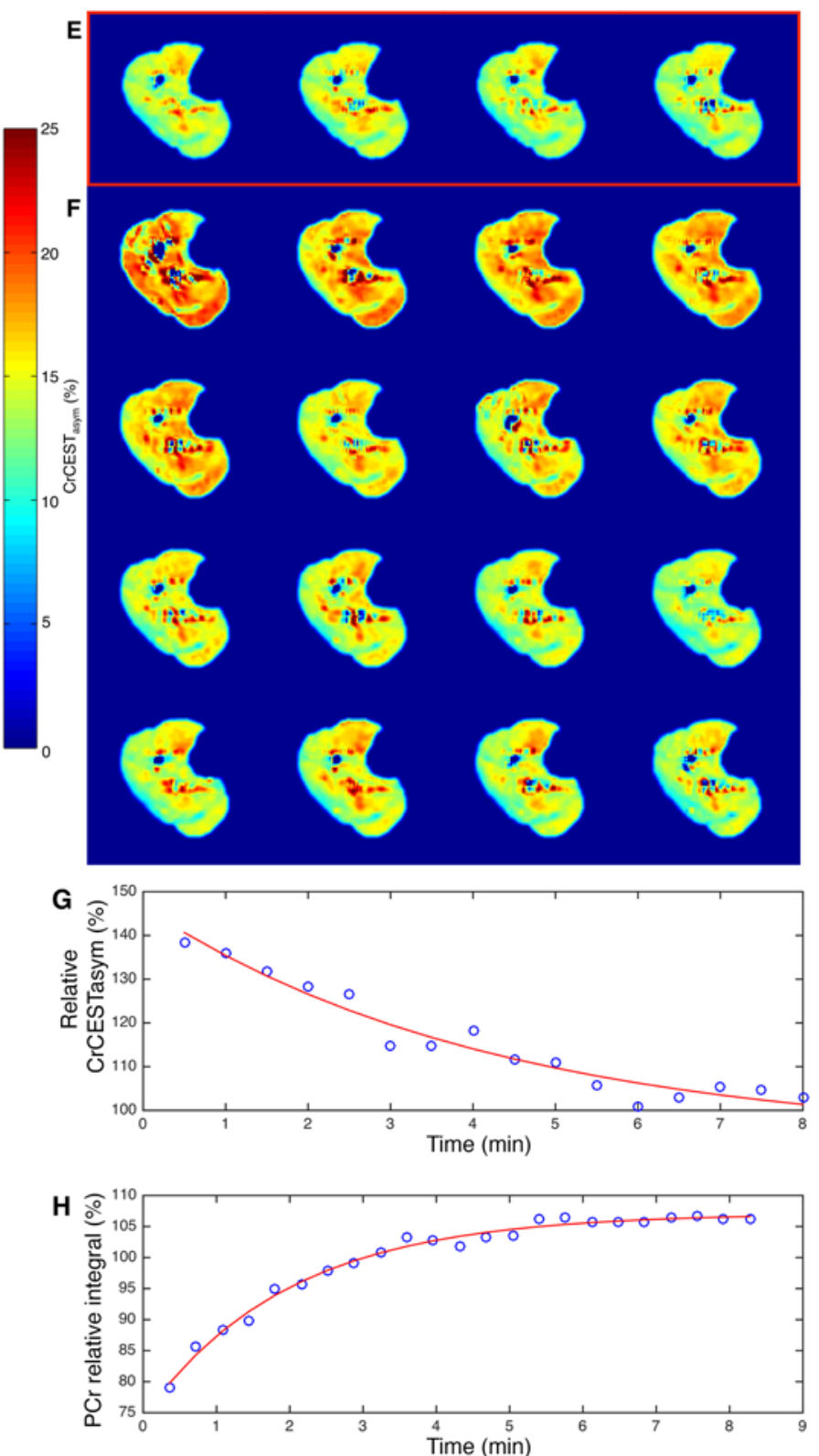

Figure 1. Example resting and postexercise CrCEST recovery images and summary curves. (A-D) (left) Images from a heathy 21-year-old female. (EH) (right) Images from a 21-year-old female with a mitochondrial DNA mutation, m.1630G >A (tRNA-Val) with hearing impairment, short stature, and stroke (53). Panels $\mathbf{A}$ and $\mathbf{E}$ show the sequential resting creatine chemical exchange saturation transfer (CrCEST) images ( 1 image every 30 seconds at rest), overlaid on the manually segmented, anatomical axial calf muscle image, for the healthy and affected individuals, respectively. The images encompass the muscle region of the right calf. The intensity of the color in each image, as shown on the color bar, is in proportion to the CrCEST percentage asymmetry signal, reflecting the amount of free creatine. Resting CrCEST appears lower in the affected individual as well (e.g., in soleus, CrCEST asymmetry is $11.1 \%$ in the affected individual versus $13.2 \%$ in healthy individual). Panels $\mathbf{B}$ and $\mathbf{F}$ show the sequential postexercise images (1 image every 30 seconds after cessation of exercise) in the healthy and affected individuals, respectively. Both subjects exercised, as indicated by the increase in free creatine, although the specific muscle groups used differ, and the percentage change in CrCEST for the same exercise was higher in the affected individual (e.g., in the soleus, $28 \%$ in the affected individual versus $10 \%$ in the healthy control). By 2 minutes after exercise, the healthy volunteer's CrCEST image resembles the baseline image, but in the affected individual, the exponential time constant for the decline in $\mathrm{Cr}$ after exercise $(\tau \mathrm{Cr})$ is prolonged (e.g., in the soleus, 5.9 minutes in the affected individual versus 1.1 minutes in the healthy control). Panels $\mathbf{C}$ and $\mathbf{G}$ show the postexercise CrCEST signal recovery summarized over the anatomic region corresponding to the approximate area of the surface ${ }^{31} \mathrm{P}-\mathrm{MRS}$ coil. Panels $\mathbf{D}$ and $\mathbf{H}$ show the postexercise phosphocreatine ( $\mathrm{PCr}$ ) signal recovery in this same anatomic region. In both modalities, prolonged postexercise recovery is observed in the affected individual relative to the healthy individual. 
Table 2. Creatine chemical exchange saturation transfer (CrCEST) MRI results

\begin{tabular}{|c|c|c|}
\hline & Mitochondrial Disease $(n=13)$ & Healthy Volunteers $(n=14)$ \\
\hline \multicolumn{3}{|c|}{ Resting CrCEST (\% asymmetry, index of free creatine concentration) } \\
\hline Medial gastrocnemius (median, IQI) & $11.5(11.1-12.4)$ & $12.1(11.7-12.7)$ \\
\hline Soleus (median, IQI) & $12.3(11.7-13.5)$ & $12.4(11.7-13.2)$ \\
\hline \multicolumn{3}{|c|}{ \% change in CrCEST with exercise (\% change from baseline) } \\
\hline Medial gastrocnemius & $32.9 \pm 15.6$ & $23.7 \pm 15.3$ \\
\hline \multicolumn{3}{|l|}{$\tau \mathrm{Cr}$ (in minutes) } \\
\hline Medial gastrocnemius (median, IQI) & $2.2^{A}(1.7-2.9)$ & $1.4(0.8-1.7)$ \\
\hline Lateral gastrocnemius (median, IQI) & $2.0(1.2-3.6)$ & $1.4(0.6-2.0)$ \\
\hline Soleus (median, IQI) & $2.1(1.7-3.7)$ & $1.7(1.1-2.7)$ \\
\hline \multicolumn{3}{|c|}{${ }^{31}$ P Magnetic Resonance Spectroscopy Parameters ${ }^{\mathrm{B}}$} \\
\hline End-exercise pH & $6.99 \pm 0.05^{A}$ & $7.04 \pm 0.06$ \\
\hline$\tau \mathbf{P C r}$ (in minutes; median, IQI) & $1.10(0.87-1.83)$ & $0.77(0.69-1.40)$ \\
\hline \multicolumn{3}{|c|}{$\begin{array}{l}{ }^{A} P<0.05 \text { for differences between subjects with mitochondrial disease versus healthy volunteers by } 2 \text {-sample } t \text { test, Wilcoxon rank-sum test, or } \\
\text { chi-square test, as appropriate. All statistically significant differences are shown in bold text. }{ }^{B} \text { Not all subjects completed }{ }^{11} \mathrm{P} \text { magnetic resonance } \\
\text { spectroscopy ( }{ }^{31} \mathrm{P}-\mathrm{MRS} \text { ) imaging given time constraints, as CrCEST was prioritized ( } n=11 \text { mitochondrial disease subjects and } n=12 \text { control subjects } \\
\text { that completed }{ }^{31} \mathrm{P}-\mathrm{MRS} \text { imaging). No attempt was made to exclude any outlier values for this summary table. Means are presented } \pm S D \text { except where } \\
\text { indicated in cases of non-normal variable distributions, in which case medians } \pm \text { IQI are shown. The Shapiro-Wilk test was used to assess normality } \\
\text { of distribution except that a normal distribution was assumed for normalized scales. IQI, interquartile interval; MET, metabolic equivalent; PCr, } \\
\text { phosphocreatine, } \mathrm{Pi} \text {, inorganic phosphate; } \tau \mathrm{PCr} \text {, exponential time constant for the decline in PCr. }\end{array}$} \\
\hline
\end{tabular}

surrounding tissue. During saturation with a specialized imaging pulse, the protons on the metabolite of interest exchange their saturated magnetization with the protons in water. The subsequent decrease in the water signal results in contrast that reflects the concentration dependence of the metabolite, with high spatial resolution. With CEST, the creatine content can be simultaneously measured in every muscle of a leg in vivo $(13,14)$. Recent studies in healthy humans have demonstrated that CEST imaging of free creatine can readily provide muscle group-specific information about OXPHOS capacity. CrCEST is used to determine the exponential time constant for the decline in $\mathrm{Cr}$ after exercise $(\tau \mathrm{Cr})$, which corresponds inversely to the exponential time constant for the recovery of PCr after exercise ( $\tau \mathrm{PCr}$ ) observed with ${ }^{31} \mathrm{P}-\mathrm{MRS}(13,14)$.

The objective of this proof-of-principle study was to determine whether CrCEST-derived measurements of postexercise exponential decline in free creatine were prolonged in individuals with primary genetic-based mitochondrial diseases, as compared with matched healthy volunteers. We sought to demonstrate the feasibility of this technique in affected individuals, and thus lay the foundation for future studies of the utility of CrCEST as a longitudinal biomarker of in vivo tissue OXPHOS capacity.

\section{Results}

Subject characteristics $(n=27)$. Characteristics are summarized in Table 1 . The 2 groups were well matched with respect to age, sex, and body mass index (BMI). Some affected individuals were nonambulatory, as reflected in overall group differences in weekly physical activity (expressed in metabolic equivalent of activity in hours [MET-hours], $P=0.049$ ) (Table 1). Results of CrCEST imaging, including resting-state CrCEST values, percentage change after exercise, and $\tau \mathrm{Cr}$; and ${ }^{31} \mathrm{P}-\mathrm{MRS}$ measurements of baseline ratio of $\mathrm{PCr}$ to inorganic phosphate $(\mathrm{PCr} / \mathrm{Pi})$, percentage change of $\mathrm{PCr}, \mathrm{pH}$, and $\tau \mathrm{PCr}$, are provided in Table 2. Diagnostic details for individuals with mitochondrial disease $(n=13)$ are provided in Table 3 . Physical activity, which is expected to impact muscle OXPHOS capacity, was included as a covariate in mixedeffects regression models (Table 4).

Determination of $\tau C r$. Figure 1 shows representative images of resting $\mathrm{CrCEST}$ levels (panels A and E), 


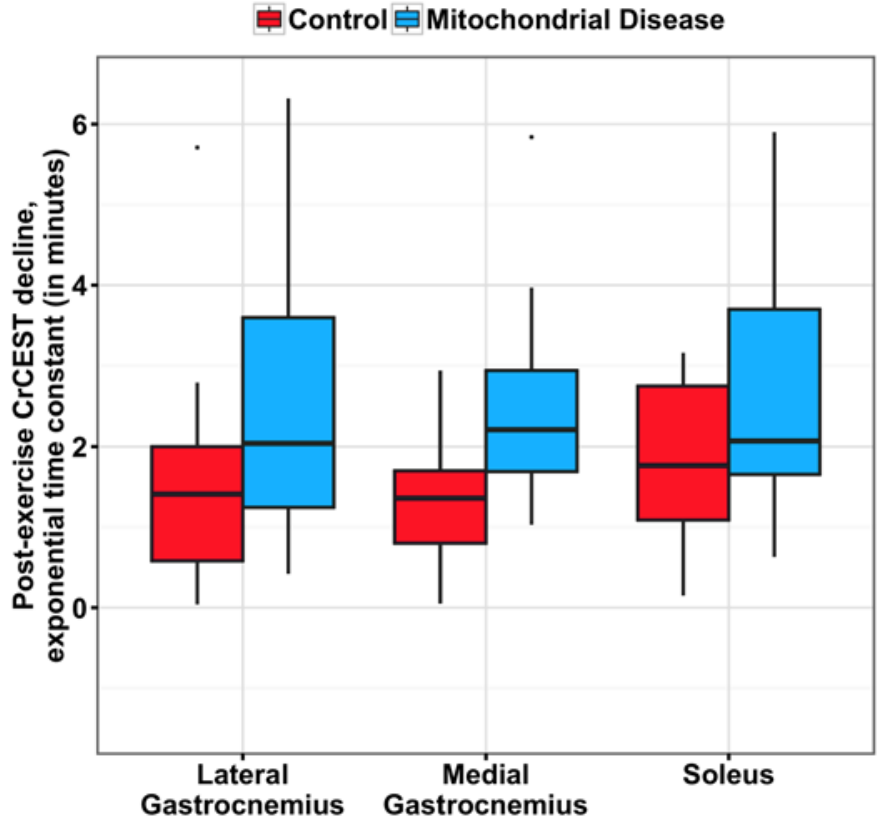

Figure 2. Box plots for postexercise creatine chemical exchange saturation transfer (CrCEST) exponential time constant $(\tau \mathrm{Cr})$, an index of skeletal muscle oxidative phosphorylation capacity, are shown (red = control, blue = mitochondrial disease). The horizontal line corresponds to the median, the lower and upper margins of the boxes correspond to the $25^{\text {th }}$ and $75^{\text {th }}$ percentiles, respectively, and whiskers show $1.5 \times$ the interquartile interval (IQI). The distribution of $\tau \mathrm{Cr}$ is not normal so nonparametric statistics are shown here. Log-transformed $\tau$ Cr values were used in statistical modeling. A prolonged $\tau$ Cr value corresponds to less oxidative phosphorylation capacity. Mitochondrial disease increases $\tau \mathrm{Cr}$ by $0.32 \mathrm{SDs}(P=0.017)$. The range of values shown results in part from the intersubject variability of which muscle groups were engaged in exercise and to what extent.

and $\tau \mathrm{Cr}$ (panels $\mathrm{B}$ and $\mathrm{F}$ ). Figure 1 also shows the corresponding postexercise CrCEST and $\mathrm{PCr}$ profiles. Overall, subjects in both groups demonstrated a measurable muscle group-specific increase (range $17.4 \%$ to $32.9 \%$ ) in CrCEST signal after exercise. $\tau \mathrm{Cr}$ was not normally distributed. Nonparametric bivariate analysis of the medial gastrocnemius (MG) (Table 2) showed a significant difference between affected individuals and controls in $\tau \mathrm{Cr}$ $(P=0.026)$. Other muscle groups were less consistently exercised in all subjects, and results for $\tau \mathrm{Cr}$ were in a similar direction, but did not achieve statistical significance. Mixed-effects regression

models of $\tau \mathrm{Cr}$ (log-transformed given non-normal distribution, Table 4) demonstrated a statistically significant effect of mitochondrial disease in all models. None of the other clinical covariates examined showed a statistically significant effect. Figure 2 is a box plot for $\tau \mathrm{Cr}$ values after exercise, which are longer in individuals with mitochondrial diseases, though an overlap is observed even after adjustment for covariates. Log-transformed $\tau \mathrm{Cr}$ values were used in statistical modeling. Mean model-derived estimates illustrate the longer $\tau \mathrm{Cr}$ for individuals with mitochondrial diseases as compared with controls $(P=0.017$ in Model 1$)$.

Resting CrCEST. Resting CrCEST, an index of free creatine concentration, showed no statistically significant differences between affected and unaffected individuals (Tables 2 and 5). With respect to muscle group, resting CrCEST, as reflected in percentage CrCEST asymmetry per unit muscle area, was highest in soleus as compared with either lateral gastrocnemius (LG) $(P=0.0058)$ or MG $(P=0.030)$ by posthoc Tukey testing in Model 1, with similar results in Models 2 and 3 (Table 5). A positive association was observed between self-reported total physical activity (MET-hours per week) and a higher resting CrCEST, but this result did not reach statistical significance in the multivariable models (standardized $\beta=0.23, P=0.070$, Table 5). Several subjects with mitochondrial myopathy had very low levels of CrCEST, hence the large variance in signal, but overall we did not observe a statistically significant difference between affected individuals and unaffected volunteers in resting CrCEST.

${ }^{31} P$-MRS. Four subjects (2 of 13 with mitochondrial disease, 2 of 14 healthy controls) completed only CrCEST imaging, and not ${ }^{31} \mathrm{P}-\mathrm{MRS}$. These participants elected not to reenter the scanner for the second same-day study. To examine the association between ${ }^{31} \mathrm{P}-\mathrm{MRS}$ and CrCEST measurements in the remaining participants, a CrCEST region of interest was defined corre-

Table 3. Diagnoses in individuals with mitochondrial respiratory chain diseases

Diagnoses $(n=13)$

Chronic progressive external ophthalmoplegia-plus C100RF12 (c.1110C>G; p.F370L), 1 subject; mitochondrial DNA deletions on muscle biopsy, 2 subjects

MFN2 (c.1699A>G; p.M567V)

POLG (c.2209G >C; p.E441G); m.13064T>C (ND5)

MELAS: m.3288A $>$ G (tRNA-Leu)

MELAS-like: m.1630A>G (tRNA-Val)

GJB1 (c.14G>T; p.G5V); VUS

SYNE1 (c.1162G>A; p.D388N, c.7066C>T; p.L2356F); VUS

Friedreich Ataxia: FXN GAA triplet-repeat expansions (2 subjects; triplet expansion lengths 454/777 and $525 / 1050$ for these subjects' FXN alleles, respectively) SDHD (c.209G>T; pR70M) SDHB (c.600G > T; pW200C)

Subjects had clinical and/or biochemical features consistent with disorders of the mitochondrial respiratory chain, and molecular diagnoses as noted. In 2 of 13 subjects, the identified mutations were variants of uncertain significance (VUS), with causality as yet to be established. 

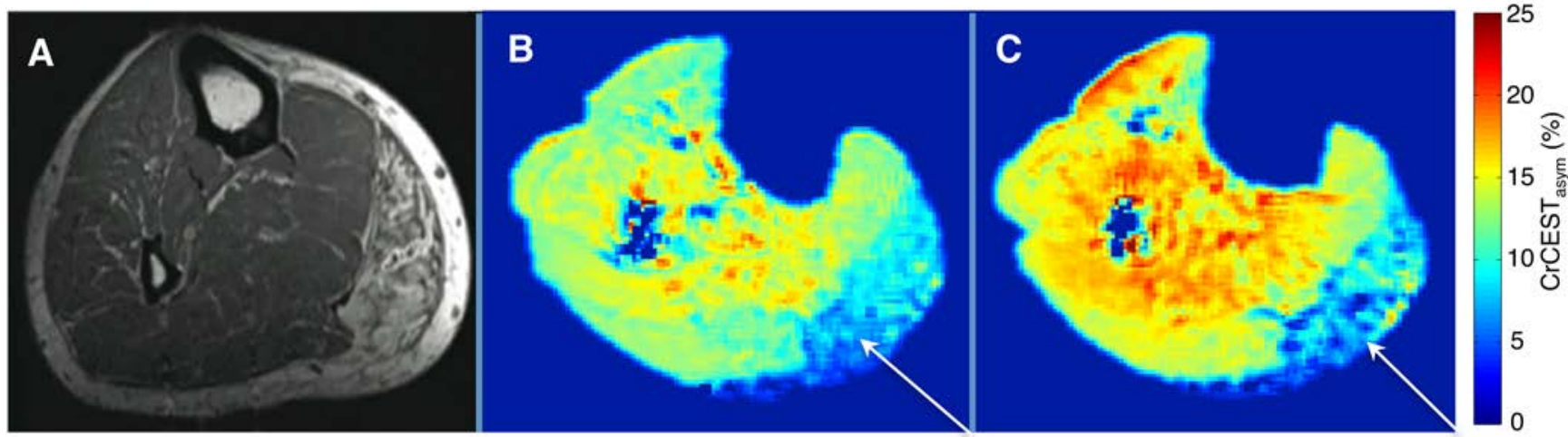

Figure 3. Example of muscle group-specific metabolic variation captured by CrCEST. Images are shown from the right leg of a 60-year-old man with chronic progressive external ophthalmoplegia due to a mutation in C100RF12 (c.1110C>G; p.F370L). (A) A 1.5T clinical image, which shows nearly complete fatty replacement of the medial gastrocnemius. (B) The corresponding area is indicated with a white arrow on a resting creatine chemical exchange saturation transfer (CrCEST) image obtained at 7.0T. The intensity of the color in each image, as shown on the color bar, is in proportion to the CrCEST percentage asymmetry signal reflecting the amount of free creatine. In B, the resting CrCEST signal is much lower in the medial gastrocnemius than in other muscle groups. After exercise, there is no increase in CrCEST signal in this region (C, white arrow).

sponding to the leg area (including portions of multiple muscle groups) assessed by the ${ }^{31} \mathrm{P}-\mathrm{MRS}$ surface coil. A positive within-subject association was observed between log-transformed $\tau \mathrm{PCr}$ and log-transformed $\tau \mathrm{Cr}$ (Pearson's correlation coefficient $=0.43, P=0.047$ ). Within-subject log-transformed resting $\mathrm{PCr} / \mathrm{Pi}$ was also positively associated with log-transformed resting CrCEST (Pearson's correlation coefficient $=$ $0.44, P=0.023)$. In addition, prior studies with ${ }^{31} \mathrm{P}-\mathrm{MRS}(15)$ have posited that reduced creatine transport into muscle and lower resting creatine may be related to postexercise PCr recovery. Indeed, we found that lower resting CrCEST (log-transformed) was associated with prolonged $\tau \mathrm{PCr}$ (log-transformed), with a Pearson's correlation coefficient of -0.42 and $P=0.046$.

While resting $\mathrm{pH}$ did not differ between mitochondrial disease subjects and unaffected controls, postexercise $\mathrm{pH}$ in the affected group was slightly lower: 6.99 versus $7.04(P<0.05$; Table 1$)$. This likely reflects the exercise-induced increased percentage change in $\mathrm{Cr}$ or $\mathrm{PCr}$ in affected individuals, though the latter changes are highly variable and do not reach statistical significance at the group level.

Anatomic variation. Figure 3 shows images from the right leg of a 60 -year-old man with chronic progressive external ophthalmoplegia due to a pathogenic autosomal dominant mutation in C10ORF2 (c.1110C>G; p.F370L). Figure 3A shows nearly complete fatty replacement of the MG. In Figure 3B, the resting CrCEST signal is much lower in the MG than in other muscle groups. After exercise, there is no increase in CrCEST signal in this region (Figure 3C, white arrow). These images illustrate the advantage of performing muscle group-specific measurements in individuals with mitochondrial diseases, given the anatomic variation that can occur in metabolic derangement (3).

Sensitivity analyses. Main analyses were repeated while excluding specific subsets of participants to ensure the robustness of the main findings. Exclusion of either participants with non-classic mitochondrial diseases, including those with succinate dehydrogenase (SDH) mutations (2 of 13) and sequence variants of uncertain significance ( 2 of 13$)$, or participants taking supplemental creatine $(n=3)$ did not substantially alter the finding of prolonged $\tau \mathrm{Cr}$ in affected individuals (effect of mitochondrial disease, standardized $\beta=$ $0.27, P=0.060$ and $\beta=0.32, P=0.027$, respectively).

Intentional exercise. Figure 4 is a correlogram showing the relationships between intentional exercise, baseline CrCEST or $\mathrm{PCr} / \mathrm{P}_{\mathrm{i}}$, and postexercise $\tau \mathrm{Cr}$ or $\tau \mathrm{PCr}$. Nonparametric bivariate correlation analyses were performed for these non-normally distributed outcomes. To allow for the possibility of muscle group differences in response to habitual intentional exercise, muscle groups were analyzed separately.

Overall, more habitual intentional exercise, whether measured in hours per week (reflecting time spent exercising) or MET-hours per week (reflecting both time and intensity of exercise) was associated with higher resting bioenergetic capacity, represented either by $\mathrm{CrCEST}$ or $\mathrm{PCr} / \mathrm{P}_{\mathrm{i}}$. This positive association between habitual intentional exercise and resting CrCEST persisted after statistical correction for multiple testing in the MG. In all of the muscle groups tested, and using both imaging modalities, more habitual intentional exercise was associated with shorter postexercise return of metabolites (free 
Table 4. Mixed-effects regression models of log-transformed postexercise CrCEST decline time constant ( $\tau \mathrm{Cr}$, in minutes), an index of skeletal muscle OXPHOS capacity, where prolonged $\tau \mathrm{Cr}$ suggests lower OXPHOS capacity

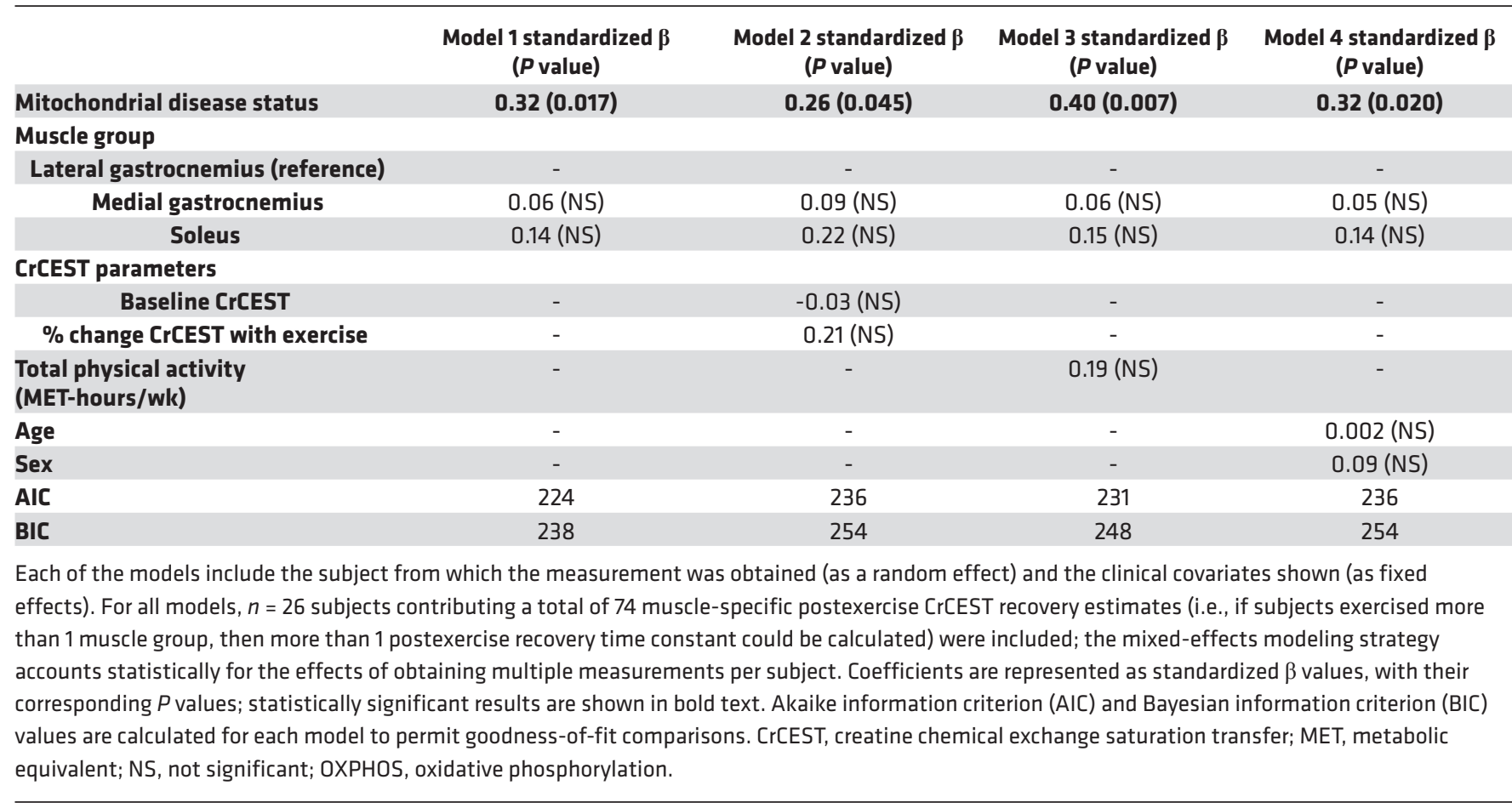

creatine, $\mathrm{PCr}$ ) to baseline, suggestive of more robust OXPHOS capacity.

In individuals with mitochondrial disease, there was a positive association between habitual exercise and baseline CrCEST, particularly in the soleus. Also, there was a positive association between habitual exercise and more rapid decline of CrCEST after exercise, suggestive of greater OXPHOS capacity, particularly in MG. In control participants, we observed a nominal association between habitual exercise and resting CrCEST in 2 muscle groups, but we did not detect an association between usual habitual exercise and imaging estimates of OXPHOS capacity. Finally, given the observed associations of imaging parameters with intentional exercise, we repeated mixed-effects regression analyses of the effects of covariates on $\tau \mathrm{Cr}$ and resting CrCEST with intentional exercise (MET-hours per week) in place of total physical activity (MET-hours per week). These results are provided in Supplemental Table 1; supplemental material available online with this article; doi:10.1172/jci.insight.88207DS1. The independent association of mitochondrial disease status with prolonged $\tau \mathrm{Cr}$ was confirmed even when habitual intentional exercise was included in the model in place of total physical activity (for effect of mitochondrial disease, standardized $\beta$ $=0.32, P=0.027$ ). Intentional exercise was independently associated with higher resting CrCEST, but this association did not achieve statistical significance (for effect of habitual intentional exercise, standardized $\beta=0.23, P=0.064)$.

Reproducibility. Results of a preliminary reproducibility study of CrCEST-based measures were obtained using a 3T whole-body scanner, and are shown in Supplemental Figures 1 and 2. In 5 healthy individuals who underwent 3T CrCEST imaging on 2 separate days within 1 week, the mean within-subject coefficient of variation (COV) for resting CrCEST percentage asymmetry, an index of free creatine concentration, was $3.4 \% \pm 1.4 \%$ (SEM) in LG, $8.2 \% \pm 1.8 \%$ in $\mathrm{MG}$, and $4.5 \% \pm 1.4 \%$ in soleus. All participants engaged LG in exercise, and most engaged MG; thus, postexercise $\tau \mathrm{Cr}$ results are shown for these 2 muscle groups. Mean within-subject COV for $\tau$ Cr was $16.0 \% \pm 7.0 \%$ (SEM) in LG and $13.5 \% \pm 6.1 \%$ in MG.

\section{Discussion}

Incorporating CrCEST in the imaging-based investigation of OXPHOS capacity in individuals with primary mitochondrial disorders yields several benefits. The CrCEST technique provides a measurement of free cre- 


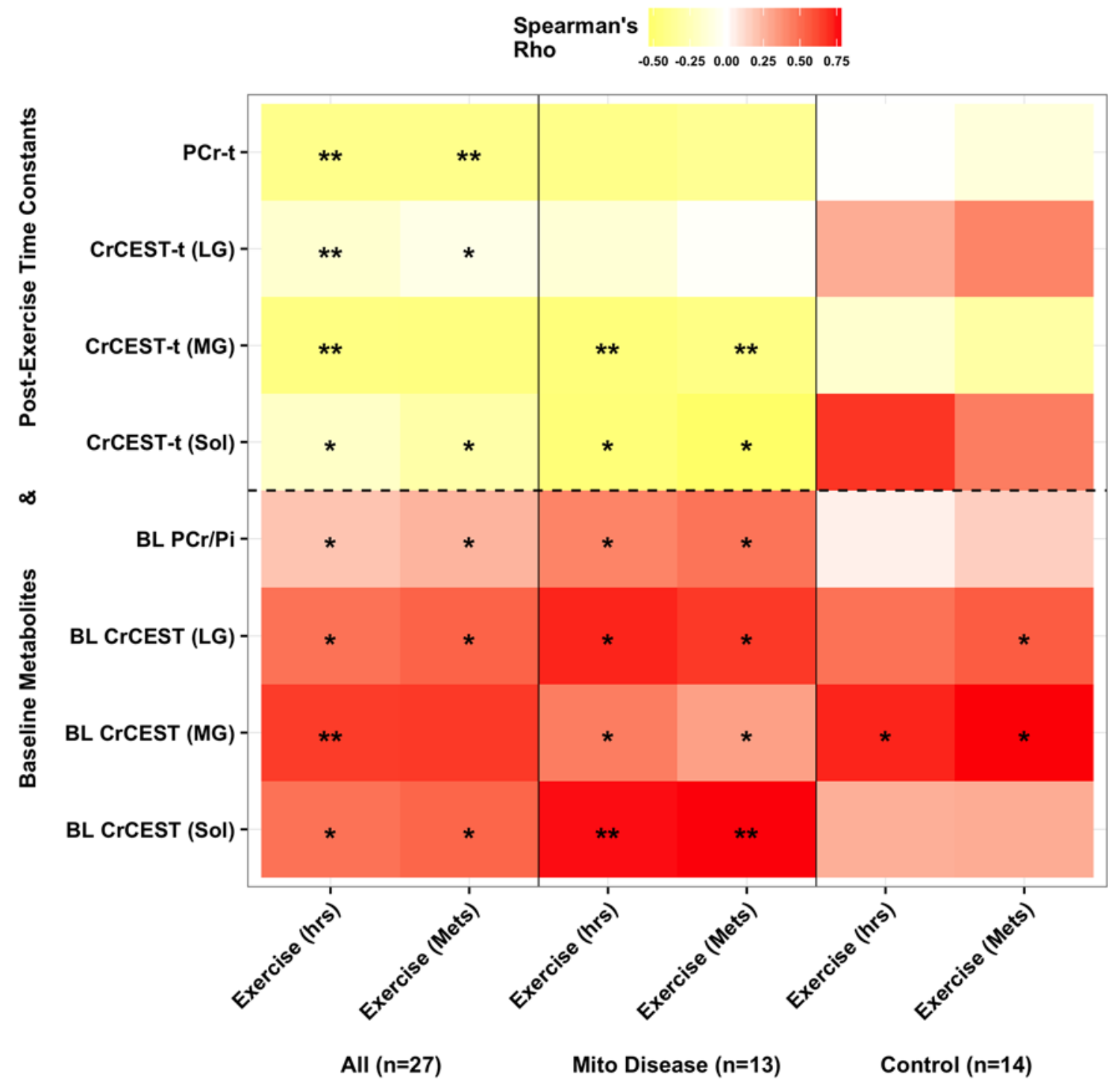

Figure 4. Correlogram of the association between intentional exercise, expressed either as hours per week (reflecting time spent exercising), or MET per week (reflecting both time and intensity spent exercising) and measured imaging parameters. The results of nonparametric correlation analyses are shown for all participants (first group of 2 columns), for participants with mitochondrial disease (second group of 2 columns), and for control participants (third group of 2 columns). Groups are separated by horizontal black lines. The bottom 4 rows indicate baseline (i.e., preexercise) metabolite concentration: baseline creatine chemical exchange saturation transfer (CrCEST) in the soleus, medial gastrocnemius, and lateral gastrocnemius, and phosphocreatine to inorganic phosphate ratio $\left(\mathrm{PCr} / \mathrm{P}_{\mathrm{i}}\right)$ in the region of interest captured by ${ }^{31} \mathrm{P}$ magnetic resonance spectroscopy $\left({ }^{31} \mathrm{P}-\mathrm{MRS}\right)$. A higher resting CrCEST or PCr/ $P_{i}$ value suggests more bioenergetic capacity at rest. The top 4 rows indicate postexercise exponential time constants for return to baseline of CrCEST in soleus, medial gastrocnemius, and lateral gastrocnemius, and PCr in the region of interest captured by ${ }^{31} \mathrm{P}-\mathrm{MRS}$. A longer postexercise time constant to return to baseline suggests decreased oxidative phosphorylation capacity. The 2 types of imaging assessment (baseline, postexercise) are separated by the dashed horizontal black line. As indicated by the color bar, positive associations are shown in red, and negative associations in yellow. ${ }^{*}$ Nominal $P$ value $<0.05$; ${ }^{* *}$ Bonferroni $P$ value $<0.05$ (adjusted for the 16 comparisons shown for each group). BL, baseline; Sol, soleus; MG, medial gastrocnemius; Mito, mitochondrial; LG, lateral gastrocnemius.

atine, while spectroscopic methods such as ${ }^{31} \mathrm{P}-\mathrm{MRS}$ and proton $\left({ }^{1} \mathrm{H}\right)$ MRS can only measure $\mathrm{PCr}$ and total creatine, respectively. The ability to measure free creatine is useful both in static measurements of resting-state skeletal muscle, as well as in dynamic exercise studies. Resting levels of free creatine may be altered in subjects with neuromuscular diseases who could potentially have reduced creatine transport into muscle $(15,16)$. As creatine monohydrate supplements are often used for therapeutic purposes in mitochondrial disorders (17), it may prove useful in future studies to estimate free creatine with CrCEST as a potential factor influencing clinical response to treatment. While we did observe low levels of resting creatine, as measured by CrCEST, in several subjects with mitochondrial myopathy, the overall group difference between affected and unaffected individuals overall did not reach statistical significance. The subjects' self-reported physical activity (MET- 
Table 5. Mixed-effects regression models of log-transformed resting CrCEST (\% asymmetry), an index of free creatine concentration, where higher CrCEST may reflect greater bioenergetic capacity at rest

\begin{tabular}{|c|c|c|c|}
\hline & Model 1 standardized $\beta$ ( $P$ value) & Model 2 standardized $\beta$ ( $P$ value) & Model 3 standardized $\beta$ ( $P$ value) \\
\hline Mitochondrial disease status & -0.16 (NS) & -0.07 (NS) & -0.17 (NS) \\
\hline \multicolumn{4}{|l|}{ Muscle group } \\
\hline Lateral gastrocnemius (reference) & - & - & - \\
\hline Medial gastrocnemius & 0.06 (NS) & 0.06 (NS) & 0.06 (NS) \\
\hline Soleus & $0.35(0.0029)$ & $0.35(0.0028)$ & $0.35(0.0031)$ \\
\hline $\begin{array}{l}\text { Total physical activity } \\
\text { (MET-hours/wk) }\end{array}$ & - & $0.23(0.07)$ & - \\
\hline Sex & - & - & 0.01 (NS) \\
\hline AIC & -72 & -62 & -52 \\
\hline BIC & -57 & -45 & -33 \\
\hline \multicolumn{4}{|c|}{$\begin{array}{l}\text { Each of the models include the subject from which the measurement was obtained (as a random effect) and the clinical covariates shown (as fixed } \\
\text { effects). For all models, } n=27 \text { subjects contributing a total of } 84 \text { muscle-specific CrCEST measurements were included; the mixed-effects modeling } \\
\text { strategy accounts statistically for the effects of obtaining multiple measurements per subject. Coefficients are represented as standardized } \beta \text { values, with } \\
\text { their corresponding } P \text { values; statistically significant results are shown in bold text. Akaike information criterion (AIC) and Bayesian information criterion } \\
\text { (BIC) values are calculated for each model to permit goodness-of-fit comparisons. CrCEST, creatine chemical exchange saturation transfer; MET, metabolic } \\
\text { equivalent; NS, not significant. }\end{array}$} \\
\hline
\end{tabular}

hours per week) appeared to be positively associated with resting CrCEST levels, but the association did not reach statistical significance in mixed-effects regression analyses including all muscle groups (standardized $\beta$ $=0.23, P=0.070$, Table 5). In bivariate correlation analyses of individual muscle groups (Figure 4), subjects' habitual intentional exercise was positively associated with higher levels of resting CrCEST and more rapid return of CrCEST to baseline after exercise in the MG. A positive association between exercise and CrCEST parameters was also evident in mitochondrial disease patients analyzed separately.

A second major advantage of CrCEST is its high spatial resolution, which provides muscle group-specific estimates of relative free creatine concentrations. The capacity to make muscle group-specific measurements is critical in studies of metabolic disorders, because different muscle groups may have distinct metabolic and contractile properties, including both mitochondrial density and the relative ratio of type I oxidative fibers to type II glycolytic fibers (3). Differences in muscle fiber type may contribute to metabolic disease; there is some evidence that the ratio of glycolytic/oxidative enzyme activities is related to insulin resistance in obese patients and some diabetic populations $(18,19)$. Also, muscle groups may respond differently to genetic or acquired mitochondrial impairment, as illustrated in Figure 3. In this subject, who had fatty replacement specific to the MG, we also observed very low resting-state CrCEST in the MG, and no subsequent increase following exercise. CrCEST may prove a useful tool to mechanistically investigate the response of specific muscle groups to exercise therapy.

Our data for all subjects, while at rest, indicates that soleus has a higher CrCEST percentage asymmetry, suggesting that this muscle has a higher concentration of free creatine than either the MG or LG. This finding might be expected given the higher proportion of oxidative fibers in soleus in previous, biopsy-based studies $(20,21)$.

After dynamic exercise, $\tau \mathrm{Cr}$ (representing the decline in CrCEST after exercise) was prolonged. Prolonged $\tau \mathrm{Cr}$ reflects decreased skeletal muscle OXPHOS capacity in individuals with primary mitochondrial diseases relative to a cohort of healthy volunteers. This statistically significant difference persisted even after accounting for other clinical covariates that could affect OXPHOS capacity. The benefits of CrCEST's high spatial resolution were also evident in the exercise component of the study, as the method provided information about all muscle groups in the exercising limb. Historically, in ${ }^{31} \mathrm{P}-\mathrm{MRS}$ exercise studies, specific muscles were targeted if the entire exercising limb could not be included in the area assessed by the coil. Unfortunately, if a particular subject did not utilize the targeted muscles, no recovery time constant could be calculated. Individuals with muscle diseases may need to use additional or alternate muscle groups to complete an exercise task. With the CrCEST technique, postexercise recovery time constants can be calculated for each muscle group that is actually deployed. 
It is interesting to note that although there were directionally consistent group-specific differences between $\tau \mathrm{Cr}$ and $\tau \mathrm{PCr}$ obtained from the 2 separate bouts of exercise, and also a positive within-subject correlation between $\tau \mathrm{Cr}$ and $\tau \mathrm{PCr}$, overall $\tau \mathrm{Cr}$ values tended to be longer in this study. We speculate that this could be attributed in part to the differences in modalities, in which the surface coil used for ${ }^{31} \mathrm{P}-\mathrm{MRS}$ provides an unlocalized signal, whereas the volume coil used for CrCEST imaging enables exact slice selection. Though a more in-depth validation than presented here is available in Kogan et al. (13), an intensive comparison of the 2 methods, in which slices for CrCEST are chosen based on the excitation profile of the surface coil, are the focus of an ongoing study. The effects of protocol design and other factors on $\tau \mathrm{Cr}$ will also be the focus of future investigation. Nonetheless, the results of our initial study further illustrate several benefits of including CrCEST in studies of skeletal muscle metabolism. CrCEST may ultimately provide insight into the aspects of the PCr shuttle that cannot be studied with ${ }^{31} \mathrm{P}-\mathrm{MRS}$ alone. Indeed, as has been previously suggested, the capacity to estimate resting creatine levels is also important in subjects with mitochondrial myopathies who may have altered creatine transport into muscle (15).

Exercise, particularly at high intensity, is one of the most potent signals for mitochondrial biogenesis $(22,23)$. Imaging studies of PCr metabolism have been used to measure the effects of exercise on bioenergetic capacity (24). To enrich our interpretation of CrCEST imaging findings, we performed detailed analyses of the association between habitual intentional exercise, captured by the validated physical activity data collection instrument (25), and the imaging parameters measured. In bivariate analyses, we demonstrated a muscle group-specific positive association between habitual intentional exercise and that both resting CrCEST and postexercise CrCEST return to baseline. In multivariate analyses where mitochondrial disease diagnosis was also included in the statistical model, the independent effects of intentional exercise did not reach statistical significance, likely the result of the larger effect of disease status. However, when participants with mitochondrial disease were analyzed separately, a positive association between intentional exercise and both resting CrCEST and $\tau \mathrm{Cr}$ was observed. This result has a basis in both clinical and epidemiologic observations. Most physicians recommend exercise therapy for individuals with mitochondrial disorders (26), based on evidence of clinical benefit in mitochondrial disease $(27,28)$ and myriad other conditions (29). The optimal exercise therapy for individuals with metabolic myopathy remains the focus of ongoing study. Intriguingly, the preferred exercise strategy may be genotype specific. For example, resistance training may decrease mutation burden in individuals with mitochondrial DNA deletions (30). We did not detect a strong statistically significant association between exercise and CrCEST parameters in control participants, perhaps related to small sample size and their overall higher level of function. Much remains to be learned, and CrCEST imaging may facilitate future research into how the nature of the intentional exercise may itself affect the chronic adaptation to training in skeletal muscle (31). In addition, CrCEST imaging may be used to explore age- and muscle group-specific effects of exercise (32).

We note that in this cross-sectional study, a causal relationship between exercise and imaging results cannot be established. An alternative explanation for our findings is that individuals with mitochondrial myopathy who have higher resting CrCEST and more rapid return to baseline of CrCEST after exercise are also more able to engage in intentional exercise. However, we found that affected individuals with mild disease (Newcastle Mitochondrial Disease Adult Scale [NMDAS] total score less than 6) engaged in an average of 1.7 MET-hours of activity per week, versus 6.1 MET-hours per week in those with moderate or severe disease ( $P=0.10$ for 2-sample $t$ test). That more severely affected individuals in our study may engage in more habitual exercise presents an intriguing possibility: individuals with worse disease who exercise may have better preserved physiology and function, and greater capacity to participate in many activities, including an exercise-based research protocol. A future exercise intervention study could explore this interesting question in detail.

This study has several important strengths and limitations. One strength is that the study groups were balanced with respect to age, sex, and adiposity (BMI). Our experimental protocol minimized several factors thought to impact muscle OXPHOS capacity (pretesting meal, prestudy exercise, and time of day). Other covariates were measured, including self-reported physical activity. Because some affected subjects were nonambulatory, they had inherently lower physical activity than otherwise matched controls, which is a potential limitation to the study. In addition, we observed a range of percentage CrCEST change in study participants after exercise with our study paradigm, which required the same number of flexions and standardized resistance for each subject. For this initial proof-of-concept study, variation in percentage CrCEST change in response to light exercise was one focus of investigation. However, to address this 
potential source of variance in $\tau \mathrm{Cr}$ measurements, percentage CrCEST was included in statistical models as a covariate. Additional studies would be further strengthened by setting exercise parameters in accordance with individual work capacity, and including the work performed by each subject as a covariate $(33,34)$. In addition, it will be useful to validate techniques for individualizing exercise intensity so as to standardize percentage change in CrCEST across participants of different abilities and levels of fitness when $\tau \mathrm{Cr}$ is prioritized as the main outcome. While we have attempted to account for appropriate covariates in this population, it is important to note that other populations may have additional factors that need consideration; for example, recovery in patients with vascular disease could be influenced by blood flow, etc. Another potential limitation of the present study is that we used self-reported exercise data for analyses. However, physical activity as assessed using the same instrument has been associated with mortality in a separate study of individuals with chronic illness (kidney disease) (29); thus, we expect a reasonable degree of correspondence between our results with both imaging parameters and clinical outcomes.

Although a complete assessment of the reproducibility of CrCEST-based measures obtained at 7T of in vivo creatine metabolism was beyond the scope of this proof-of-principle study, we performed an initial analysis in healthy individuals at 3T. This pilot study was performed at 3T because MRI scanners at this field strength are more widely available, and thus may be used for future, larger studies and/or adapted for clinical use. In addition, the feasibility of CrCEST imaging at 3T has already been demonstrated (14). As this separate study reflected a sample of convenience, it did not include standardized clinical factors including fasting status, previous exercise, or time of day. For this reason, the resulting reproducibility estimates represent the lower bound of what would be achievable with careful standardization of preanalytic factors in future CrCEST studies. Even without preanalytic standardization of clinical factors and with the lower MRI field strength, we observed that a reasonable degree of consistency is achievable.

Future studies may also incorporate an investigation of the relationship between $\tau \mathrm{Cr}$ and the results of muscle biopsy studies. However, in the present study, most of the affected participants had not undergone invasive muscle biopsy for clinical purposes. Muscle biopsy had not been recommended because the clinical features, together with molecular genetic testing results, were sufficient to establish the diagnosis of mitochondrial disease without invasive biopsy testing requiring general anesthetic that may be poorly tolerated in individuals with mitochondrial disease. Affected individuals in this study with classical mitochondrial disease syndromes (chronic progressive external ophthalmoplegia-plus [CPEO-plus]; mitochondrial encephalomyopathy, lactic acidosis, and stroke-like episodes [MELAS]; MELAS-like; Friedrich's ataxia [FA]) and related genetic defects (mutations in C10orf12, POLG, MFN2, FXN) had clinical evidence of impaired muscle mitochondrial function. Deficits in mitochondrial electron transport chain activity via ex vivo skeletal muscle biopsy studies have been identified previously in each of these mitochondrial disease syndromes. For example, the gene C10orf12 encodes Twinkle, a helicase that is critical for mitochondrial DNA replication (35). Mutations in C10orf12, as well as the mitochondrial replicase, POLG, are associated with mitochondrial DNA depletion and/or deletions with muscle biopsy findings typically involving multiple respiratory chain complex enzyme deficiency and immunohistochemical evidence of cytochrome oxidase (complex IV)-deficient muscle fibers (36). In individuals with myopathy related to MELAS, skeletal muscle biopsy may demonstrate the presence of ragged red fibers, indicative of mitochondrial proliferation (37). Seminal studies are additionally reviewed in (38). In FA, abnormal muscle OXPHOS capacity is related to mitochondrial iron overload; decreased OXPHOS capacity in FA has been demonstrated by skeletal muscle biopsy (39) as well as ${ }^{31} \mathrm{P}-\mathrm{MRS}$ (40). Mutations in nonclassical mitochondrial disease genes, including SDH subunit genes have been also associated with reduced OXPHOS capacity in muscle (41, 42), although the clinical association is less clear. For this reason, in the present study we performed sensitivity analyses on the effects of excluding individuals with SDH mutations and individuals with novel gene variants of uncertain significance with respect to mitochondrial disease on our main analysis. We demonstrated persistent differences in postexercise $\tau \mathrm{Cr}$ as compared with healthy volunteers.

Heterogeneity of clinical and molecular diagnoses in affected subjects is a source of biological variance. Despite this heterogeneity, we still observed a statistically significant effect of disease status on postexercise $\tau \mathrm{Cr}$. This result shows that CrCEST imaging can identify decreased OXPHOS capacity in a genetically heterogeneous population whose molecular gene defects are likely to share a common pathophysiology involving decreased OXPHOS capacity (43). Taken together, these studies demonstrate that noninvasive CrCEST imaging can be used to quantitatively monitor physiologic changes after exercise, including both creatine content and metabolic capacity, in individuals with mitochondrial diseases. This technique has enhanced 
spatial resolution compared with traditional spectroscopy methods, and can be performed with standard ${ }^{1} \mathrm{H}$ coils, whereas ${ }^{31} \mathrm{P}-\mathrm{MRS}$ techniques require multinuclear MRI scanner hardware that may preclude more widespread use. Optimally, CrCEST will be used to complement existing ${ }^{31} \mathrm{P}-\mathrm{MRS}$-based assessments to perform a more complete, comprehensive assessment of PCr shuttle function in individuals with mitochondrial disorders. Our work lays a rational foundation to develop CrCEST as a potential noninvasive imagingbased biomarker of OXPHOS capacity that may be useful in the diagnosis, long-term monitoring, and/or evaluation of response to clinical treatments in individuals with diverse types of mitochondrial diseases.

\section{Methods}

Design and subjects. Individuals (18-65 years, inclusive) with a clinical diagnosis of a disorder of the mitochondrial respiratory chain were recruited. We prioritized eligible subjects with disorders confirmed to be caused by pathogenic mitochondrial DNA or nuclear DNA mutations affecting subunits or assembly of mitochondrial respiratory chain complexes that are associated with known clinical/pathological features. These disorders include CPEO; Kearns-Sayre syndrome; MELAS; mitochondrial encephalopathy and ragged red fibers (MERRF); neuropathy, ataxia and retinitis pigmentosa (NARP); or Leigh syndrome (44). Individuals affected with FA were also studied. FA is a mitochondrial disease in which ATP production is reduced due to GAA triplet-repeat expansions in frataxin, a mitochondrial protein involved in the formation of iron-sulfur clusters necessary for respiratory chain complex function (45). Finally, individuals were studied who had genetic deficiency of isoforms of SDH, which serves as respiratory chain complex II $(42,46)$.

Although collectively these subjects are heterogeneous with respect to their specific molecular diagnoses, the functional consequence of their defects is expected to include a deficiency in skeletal muscle OXPHOS capacity. Because multiple studies have used ${ }^{31} \mathrm{P}-\mathrm{MRS}$ to demonstrate impaired skeletal muscle OXPHOS in individuals with type 2 diabetes mellitus (47), individuals with diabetes mellitus were excluded, and fasting blood glucose hemoglobin A1c (HgbA1c) was measured on the day of the study to verify diabetes status. Healthy, nondiabetic volunteers were recruited to generate a cohort that was balanced compared with the group of affected individuals with respect to age, sex, and BMI.

Additional metrics. Height and weight were measured according to standard procedures. Blood specimens were collected after an overnight fast and processed using standard techniques for glucose, lipid pan$\mathrm{el}$, and $\mathrm{HgbA1c}$. Several validated instruments were used to measure important potential covariates affecting mitochondrial function, including self-reported physical activity, using the Chronic Renal Insufficiency Cohort (CRIC) Physical Activity Questionnaire (25), mitochondrial disease severity, using the NMDAS (43), and physical function and overall health, using the NIH Patient-Reported Outcomes Measurement Information System (PROMIS) Global Health and Physical Function Scales (48).

MRI and MRS. CrCEST images were acquired on a 7T whole-body scanner (Siemens Medical Systems). Imaging experiments were performed using a 28-channel ${ }^{1} \mathrm{H}$ knee coil. Anatomical, axial images were acquired of the right calf in all subjects to be used for segmentation (21). CrCEST imaging parameters were as follows: saturation pulse $=500 \mathrm{~ms}, \mathrm{~B} 1_{\mathrm{rms}}=123 \mathrm{~Hz}(2.9 \mu \mathrm{T})$, slice thickness $=10 \mathrm{~mm}$, flip angle $=10^{\circ}, \mathrm{TR}=6.0 \mathrm{~ms}, \mathrm{TE}=2.9 \mathrm{~ms}$, field of view $=140 \times 140 \mathrm{~mm}^{2}$, matrix size $=128 \times 128$. Four baseline images were acquired over 2 minutes with a temporal resolution of 30 seconds, followed by 2 minutes of mild plantar flexion exercise. Exercise was performed in the magnet using an MR-compatible, pneumatically controlled foot pedal, with pressure held constant at 7.5 psi for all subjects. The subjects were instructed to fully depress the ergometer pedal at a constant rate of 90 flexions over 2 minutes (1 flexion every $\sim 1.3$ seconds). Adherence was assured by visually confirming pedal depression via a direct readout of a dynamometer. In this initial proof-of-principle study, we did not attempt to vary exercise intensity according to each participant's capacity. Rather, percentage change in CrCEST in response to this light, standardized exercise workload was an important outcome of interest assessed in affected individuals as compared with controls. To test for the potential effects of differential exercise intensity on $\tau \mathrm{Cr}$, percentage change in CrCEST was included as a covariate in statistical models.

Immediately after exercise, 8 minutes of CrCEST images were acquired with the same 30 -second resolution (16 consecutive images). Image processing was performed using in-house MATLAB scripts (49, 50). Water saturation shift referencing (51) maps and $B_{1}$ maps were acquired before and after exercise, and used to generate corrected CrCEST images as described for previous studies $(14,50)$. CrCEST contrast was computed by subtracting the normalized magnetization signal at the creatine proton frequency 
$(\Delta \omega=+1.8 \mathrm{ppm})$, from the magnetization at the corresponding reference frequency on the opposite side of the water resonance $(-\Delta \omega)(52)$. CrCEST changes in individual muscle groups before and after exercise were determined by overlaying CrCEST maps onto manually segmented anatomic images. $\tau \mathrm{Cr}$ was calculated by fitting the postexercise CrCEST contrast decay, from each segmented muscle, to an exponential (50), as illustrated in Figure 1.

${ }^{31} \mathrm{P}-\mathrm{MRS}$ was performed with a $7-\mathrm{cm}$ diameter ${ }^{1} \mathrm{H} /{ }^{31} \mathrm{P}$ dual-tuned surface coil using an unlocalized free induction decay (FID) sequence: number of points $=512$, averages $=5$, and $\mathrm{TR}=2.4$ seconds, with 4 dummy scans, in order to obtain similar time resolution to the CrCEST image acquisition. ${ }^{31} \mathrm{P}-\mathrm{MRS}$ spectra were phased and baseline corrected and fitted using nonlinear least squares method with Lorentzian functions. $\tau$ PCr was determined by calculating the area under the curve for the PCr peak from every FID after exercise, and fitting the recovery to an exponential equation, as illustrated in Figure 1.

Reproducibility. A pilot reproducibility study was performed on a 3T whole-body scanner (Siemens Medical Systems), with a 15-channel knee coil. Imaging parameters were as described above, but with the optimized CEST parameters for creatine at 3T, as determined by Kogan et al. (14). Five healthy individuals were scanned on 2 separate days within 1 week. Unlike the main protocol, for this initial study of reproducibility care was not taken to standardize important clinical factors including fasting status, previous exercise, or time of day. We assessed the degree of agreement between (a) resting, preexercise CrCEST percentage asymmetry (in MG, LG, and soleus and (b) postexercise $\tau \mathrm{Cr}$.

Statistics. Clinical characteristics were summarized with standard descriptive statistics and compared between affected and unaffected individuals using parametric or nonparametric methods, as appropriate given the variable distributions. Pearson's correlation coefficient was used to measure the correlation between continuous variables. $\tau \mathrm{Cr}$ was log-transformed due to non-normal distribution, and linear mixed-effects regression analyses were performed to assess the effect of mitochondrial disease status on $\tau \mathrm{Cr}$ measured over time, adjusted for other clinical covariates in separate statistical models. These statistical models account for within-subject correlation due to repeated measures by including a subject-specific random effect. In each model, mitochondrial disease status and muscle group analyzed are included as fixed effects along with others as follows: in Model 1, no additional fixed effects, i.e., mitochondrial disease status and muscle group alone; in Model 2, mitochondrial disease status, muscle group, resting CrCEST, and percentage change in CrCEST; in Model 3, mitochondrial disease status, muscle group, and total self-reported total physical activity (MET-hours per week); and in Model 4 , mitochondrial disease status, muscle group, age, and sex.

The independent effects of clinical covariates on log-transformed resting CrCEST were also assessed using mixed-effects regression analysis. Included in these models were between-subject variability (random effect), mitochondrial disease status, and muscle group (fixed effects). As additional fixed effects, the following were included: Model 1, no additional factors, i.e., mitochondrial disease status and muscle group alone; in Model 2, mitochondrial disease status, muscle group and, total physical activity (MET-hours per week); and in Model 3, mitochondrial disease status, muscle group, age, and sex. All of the clinical covariates were considered as fixed effects in separate models as shown to avoid overfitting given the sample size and number of observations in this study. For physical activity, total physical activity (MET-hours per week) was chosen because of the statistically significant differences observed between affected individuals and healthy volunteers on this covariate. Akaike information criterion (AIC) and Bayesian information criterion (BIC) values were calculated for each model to permit goodness-of-fit comparisons. Sensitivity analyses were performed by analyzing subsets of participants with established genetic diagnoses (i.e., excluding the 2 of 13 individuals with variants of uncertain significance), and also the subset of subjects not taking creatine (i.e., excluding the 3 of 13 subjects with mitochondrial disease who were taking supplemental creatine), to assess for any potential associated source of bias. For analyses of the association between habitual intentional exercise, collected via validated instrument, with imaging parameters, nonparametric correlation analyses were performed on non-normally distributed variables, stratified by (a) group (all participants, participants with mitochondrial disease, participants without mitochondrial disease); (b) imaging technique (CrCEST, ${ }^{31} \mathrm{P}-\mathrm{MRS}$ ), and (c) parameter type (baseline, postexercise decline, or recovery time constant). Both nominal and Bonferroniadjusted statistical significance thresholds are shown. Since we observed an association between intentional exercise and both baseline and postexercise CrCEST values, we also performed a sensitivity analysis, repeating mixed-effects regression analyses with intentional exercise (MET-hours per week) in place of total physical activity (MET-hours per week) in the models to determine the independence of effects. For preliminary 
reproducibility studies, the within-subject COV between day 1 and day 2 measurements was calculated for each participant. All analyses were conducted using R (version 3.1.3), and statistical significance was taken as 2 -sided $P$ value of $<0.05$.

Study approval. This cross-sectional, observational study enrolling both cases and controls was performed under an approved Institutional Review Board protocol of the University of Pennsylvania (www.clinicaltrials.gov: NCT02154711) and was conducted according to the Declaration of Helsinki. Written, informed consent was obtained from all participants prior to their inclusion in the study.

\section{Author contributions}

SEM, MJF, RR, VAS, and CD designed the study. CD, RPRN, NW, KD, ME, HH, FY, KW, SN, DW, CPS, and EM performed the study procedures. CD, NW, HH, FY, CPS, and RX analyzed the data. CD and SEM wrote the manuscript. All authors reviewed the manuscript.

\section{Acknowledgments}

Sources of support: Diabetes Research Center at the University of Pennsylvania Pilot \& Feasibility Award from a grant sponsored by NIH DK 19525 (to SEM), NIH K12DK094723/NIH K23DK102659 (to SEM), Pediatric Endocrine Society Clinical Scholar Award (to SEM), NIH R03-DK082446 (to MJF), NIH P41EB015893 (to UPenn CMROI), NIH UL1TR000003 (to UPenn CTRC).

We thank our volunteers for their participation in research; Jacqui Meeks, Colleen Muraresku, Cassandra Strawser for their additional support; as well as the staff of the University of Pennsylvania CTRC.

Address correspondence to: Shana E. McCormack, 3401 Civic Center Boulevard, Division of Endocrinology and Diabetes, Suite 11NW, Philadelphia, Pennsylvania 19104, USA. Phone: 215.590.3174; E-mail: mccormacks1@email.chop.edu.

1. Wallace DC. A mitochondrial bioenergetic etiology of disease. J Clin Invest. 2013;123(4):1405-1412.

2. Andreu AL, et al. Exercise intolerance due to mutations in the cytochrome b gene of mitochondrial DNA. N Engl J Med. 1999;341(14):1037-1044.

3. Zierath JR, Hawley JA. Skeletal muscle fiber type: influence on contractile and metabolic properties. PLoS Biol. 2004;2(10):e348.

4. Shoffner JM, Lott MT, Wallace DC. MERRF: a model disease for understanding the principles of mitochondrial genetics. Rev Neurol (Paris). 1991;147(6-7):431-435.

5. Proctor DN, Sinning WE, Walro JM, Sieck GC, Lemon PW. Oxidative capacity of human muscle fiber types: effects of age and training status. J Appl Physiol. 1995;78(6):2033-2038.

6. Horiuchi M, Fadel PJ, Ogoh S. Differential effect of sympathetic activation on tissue oxygenation in gastrocnemius and soleus muscles during exercise in humans. Exp Physiol. 2014;99(2):348-358.

7. Chance B, Bank W. Genetic disease of mitochondrial function evaluated by NMR and NIR spectroscopy of skeletal tissue. Biochim Biophys Acta. 1995;1271(1):7-14.

8. Matthews PM, Allaire C, Shoubridge EA, Karpati G, Carpenter S, Arnold DL. In vivo muscle magnetic resonance spectroscopy in the clinical investigation of mitochondrial disease. Neurology. 1991;41(1):114-120.

9. Arnold DL, Taylor DJ, Radda GK. Investigation of human mitochondrial myopathies by phosphorus magnetic resonance spectroscopy. Ann Neurol. 1985;18(2):189-196.

10. Penn AM, et al. MELAS syndrome with mitochondrial tRNA(Leu)(UUR) mutation: correlation of clinical state, nerve conduction, and muscle ${ }^{31} \mathrm{P}$ magnetic resonance spectroscopy during treatment with nicotinamide and riboflavin. Neurology. 1992;42(11):2147-2152.

11. Parasoglou P, Xia D, Chang G, Regatte RR. Dynamic three-dimensional imaging of phosphocreatine recovery kinetics in the human lower leg muscles at 3T and 7T: a preliminary study. NMR Biomed. 2013;26(3):348-356.

12. Haris M, et al. Exchange rates of creatine kinase metabolites: feasibility of imaging creatine by chemical exchange saturation transfer MRI. NMR Biomed. 2012;25(11):1305-1309.

13. Kogan F, Hariharan H, Reddy R. Chemical exchange saturation transfer (CEST) imaging: description of technique and potential clinical applications. Curr Radiol Rep. 2013;1(2):102-114.

14. Kogan F, et al. In vivo chemical exchange saturation transfer imaging of creatine (CrCEST) in skeletal muscle at 3T. JMagn Reson Imaging. 2014;40(3):596-602.

15. Tarnopolsky MA, Parise G. Direct measurement of high-energy phosphate compounds in patients with neuromuscular disease. Muscle Nerve. 1999;22(9):1228-1233.

16. Tarnopolsky MA, Parshad A, Walzel B, Schlattner U, Wallimann T. Creatine transporter and mitochondrial creatine kinase protein content in myopathies. Muscle Nerve. 2001;24(5):682-688.

17. Parikh S, et al. A modern approach to the treatment of mitochondrial disease. Curr Treat Options Neurol. 2009;11(6):414-430.

18. Simoneau JA, Kelley DE. Altered glycolytic and oxidative capacities of skeletal muscle contribute to insulin resistance in 
NIDDM. J Appl Physiol. 1997;83(1):166-171.

19. Simoneau JA, Colberg SR, Thaete FL, Kelley DE. Skeletal muscle glycolytic and oxidative enzyme capacities are determinants of insulin sensitivity and muscle composition in obese women. FASEB J. 1995;9(2):273-278.

20. Johnson MA, Polgar J, Weightman D, Appleton D. Data on the distribution of fibre types in thirty-six human muscles. An autopsy study. J Neurol Sci. 1973;18(1):111-129.

21. Trappe SW, Trappe TA, Lee GA, Costill DL. Calf muscle strength in humans. Int J Sports Med. 2001;22(3):186-191.

22. Little JP, Safdar A, Bishop D, Tarnopolsky MA, Gibala MJ. An acute bout of high-intensity interval training increases the nuclear abundance of PGC-1 $\alpha$ and activates mitochondrial biogenesis in human skeletal muscle. Am J Physiol Regul Integr Comp Physiol. 2011;300(6):R1303-R1310.

23. Little JP, et al. Low-volume high-intensity interval training reduces hyperglycemia and increases muscle mitochondrial capacity in patients with type 2 diabetes. J Appl Physiol. 2011;111(6):1554-1560.

24. Kent-Braun JA, McCully KK, Chance B. Metabolic effects of training in humans: a 31P-MRS study. J Appl Physiol. 1990;69(3):1165-1170.

25. Feldman HI, et al. The Chronic Renal Insufficiency Cohort (CRIC) Study: Design and Methods. J Am Soc Nephrol. 2003;14(7 Suppl 2):S148-S153.

26. Parikh S, et al. Diagnosis and management of mitochondrial disease: a consensus statement from the Mitochondrial Medicine Society. Genet Med. 2015;17(9):689-701.

27. Jeppesen TD, et al. Short- and long-term effects of endurance training in patients with mitochondrial myopathy. Eur J Neurol. 2009;16(12):1336-1339.

28. Murphy JL, et al. Resistance training in patients with single, large-scale deletions of mitochondrial DNA. Brain. 2008;131(Pt 11):2832-2840.

29. Ricardo AC, et al. Healthy lifestyle and risk of kidney disease progression, atherosclerotic events, and death in CKD: findings from the Chronic Renal Insufficiency Cohort (CRIC) Study. Am J Kidney Dis. 2015;65(3):412-424.

30. Tarnopolsky MA. Exercise as a therapeutic strategy for primary mitochondrial cytopathies. J Child Neurol. 2014;29(9):1225-1234.

31. Cochran AJ, et al. Intermittent and continuous high-intensity exercise training induce similar acute but different chronic muscle adaptations. Exp Physiol. 2014;99(5):782-791.

32. Larsen RG, Callahan DM, Foulis SA, Kent-Braun JA. Age-related changes in oxidative capacity differ between locomotory muscles and are associated with physical activity behavior. Appl Physiol Nutr Metab. 2012;37(1):88-99.

33. Sleigh A, et al. Simple and effective exercise design for assessing in vivo mitochondrial function in clinical applications using P-31 magnetic resonance spectroscopy. Scientific Reports. 2016.

34. Cree-Green M, et al. Method for controlled mitochondrial perturbation during phosphorus MRS in children. Med Sci Sports Exerc. 2014;46(10):2030-2036.

35. Spelbrink JN, et al. Human mitochondrial DNA deletions associated with mutations in the gene encoding Twinkle, a phage T7 gene 4-like protein localized in mitochondria. Nat Genet. 2001;28(3):223-231.

36. Zierz CM, Joshi PR, Zierz S. Frequencies of myohistological mitochondrial changes in patients with mitochondrial DNA deletions and the common m.3243A $>$ G point mutation. Neuropathology. 2015;35(2):130-136.

37. Milone M, Klassen BT, Landsverk ML, Haas RH, Wong LJ. Orthostatic tremor, progressive external ophthalmoplegia, and Twinkle. JAMA Neurol. 2013;70(11):1429-1431.

38. DiMauro S, Bonilla E, Zeviani M, Nakagawa M, DeVivo DC. Mitochondrial myopathies. Ann Neurol. 1985;17(6):521-538.

39. Bayot A, Santos R, Camadro JM, Rustin P. Friedreich's ataxia: the vicious circle hypothesis revisited. BMC Med. 2011;9:112.

40. Lodi R, et al. Deficit of in vivo mitochondrial ATP production in patients with Friedreich ataxia. Proc Natl Acad Sci U S A. 1999;96(20):11492-11495.

41. Alston CL, et al. Recessive germline SDHA and SDHB mutations causing leukodystrophy and isolated mitochondrial complex II deficiency. J Med Genet. 2012;49(9):569-577.

42. Alston CL, et al. A recessive homozygous p.Asp92Gly SDHD mutation causes prenatal cardiomyopathy and a severe mitochondrial complex II deficiency. Hum Genet. 2015;134(8):869-879.

43. Bates MG, et al. Concentric hypertrophic remodelling and subendocardial dysfunction in mitochondrial DNA point mutation carriers. Eur Heart J Cardiovasc Imaging. 2013;14(7):650-658.

44. Stacpoole PW, et al. Design and implementation of the first randomized controlled trial of coenzyme $\mathrm{CoQ}_{10}$ in children with primary mitochondrial diseases. Mitochondrion. 2012;12(6):623-629.

45. Kaplan J. Friedreich's ataxia is a mitochondrial disorder. Proc Natl Acad Sci U S A. 1999;96(20):10948-10949.

46. Jackson CB, et al. Mutations in SDHD lead to autosomal recessive encephalomyopathy and isolated mitochondrial complex II deficiency. J Med Genet. 2014;51(3):170-175.

47. Scheuermann-Freestone M, et al. Abnormal cardiac and skeletal muscle energy metabolism in patients with type 2 diabetes. Circulation. 2003;107(24):3040-3046.

48. Barile JP, et al. Monitoring population health for Healthy People 2020: evaluation of the NIH PROMIS Global Health, CDC Healthy Days, and satisfaction with life instruments. Qual Life Res. 2013;22(6):1201-1211.

49. Kogan F, et al. Method for high-resolution imaging of creatine in vivo using chemical exchange saturation transfer. Magn Reson Med. 2014;71(1):164-172.

50. Wilson NE. ExerciseCEST. Zenodo. http://doi.org/10.5281/zenodo.59640. Published August 4, 2016. Accessed October 3, 2016.

51. Kim M, Gillen J, Landman BA, Zhou J, van Zij1 PC. Water saturation shift referencing (WASSR) for chemical exchange saturation transfer (CEST) experiments. Magn Reson Med. 2009;61(6):1441-1450.

52. Haris M, et al. A technique for in vivo mapping of myocardial creatine kinase metabolism. Nat Med. 2014;20(2):209-214.

53. Glatz C, D'Aco K, Smith S, Sondheimer N. Mutation in the mitochondrial tRNA(Val) causes mitochondrial encephalopathy, lactic acidosis and stroke-like episodes. Mitochondrion. 2011;11(4):615-619. 